\title{
Characteristics of aquaporin 1, 3, and 5 expression during early murine salivary gland development
}

\section{Fernanda de Paula ${ }^{1}$, Abigail S. Tucker ${ }^{2}$, Tathyane Harumi N. Teshima ${ }^{3}$, Milena Monteiro de

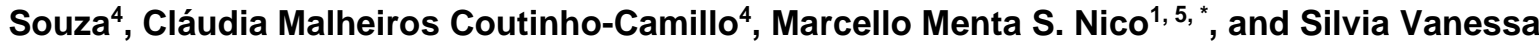 Lourenço1, 6}

1 General Pathology Department, Dental School, University of Sao Paulo, Av Prof Lineu Prestes, 2227 Cidade Universitária, São Paulo, SP CEP 05508-000, Brazil

2 Centre for Craniofacial and Regenerative Biology, King's College London, London, UK

UK

3 NIHR Clinical Lecturer in Oral Medicine, UCL Eastman Dental Institute, 21 University Street, London, Brazil

4 International Research Center, A.C.Camargo Cancer Center, Rua Taguá 440, São Paulo 01508-010,

5 Dermatology Department, Medical School, University of São Paulo, Av. Dr. Enéas de Carvalho Aguiar, 500, São Paulo, SP, Brazil

6 LIM06, Institute of Tropical Medicine, University of São Paulo, Av. Dr. Enéas de Carvalho Aguiar, 500, São Paulo, SP, Brazil

* Correspondence: mentanico@hotmail.com (M.M.S.N.); Tel.: +55 1132889935 (M.M.S.N.)

\section{ABSTRACT}

Aquaporins (AQPs) are essential to coordinate the transit of water and ions through the cell membrane. In salivary glands (SGs), AQPs have been associated with saliva formation, facilitating water absorption through the epithelium during the formation of hypotonic saliva, which is then secreted into the oral cavity. Different members of the AQP family have been suggested to play distinct roles during embryonic development, highlighted by their specific expression patterns. Here, we have investigated the expression patterns of AQP-1, AQP-3, and AQP-5 by immunofluorescence at key stages of salivary gland development, utilising cultured mouse embryonic submandibular (SMG) and sublingual (SLG) glands. The expression of AQPs was compared to a mitotic marker, phospho-histone 3 (PH3), a myoepithelial marker, smooth muscle actin (SMA), and a vascular marker, CD31. Qualitative analysis revealed that AQP-1 and AQP-3 were primarily expressed during the earlier phases of SG morphogenesis and were associated with cells undergoing mitotic processes (PH3positive). AQP-5, in contrast, was not associated to mitotic figures, but was predominantly expressed during late stages of SG morphogenesis. Our results highlight that AQPs are expressed from early stages of SG morphogenesis and exhibit complimentary expression patterns that may contribute to the morphogenesis of salivary glands.

Keywords: salivary gland, development, expression, aquaporin, mitosis 


\section{INTRODUCTION}

Aquaporins (AQPs) are a family of water channel proteins, consisting of 14 members (Chauvigné et al., 2019). AQPs are considered to be paramount in the orchestration of cellular physiological processes, as they coordinate the transit of water and ions through the cell membrane. AQPs have been especially well characterised in tubular organs, such as blood vessels, kidneys, and salivary glands (SGs), but they can be expressed in multiple organs in the human body. Each AQP member has been suggested to play distinct roles during embryonic development highlighted by the distinct expression patterns observed in many tissues (Qin et al., 2019). Several studies have described AQP-1, -3, -4 , and -5 as being the most important AQPs for SG function; however, other AQPs have been increasingly implicated in glandular physiology, including AQP-6, -7, -8, -9, and -11. (Gresz et al., 2001, Wang et al., 2003; Yang et al., 2005, Welner et al., 2006; Larsen at al., 2009; Aure et al., 2014).

AQP-1 is widely expressed in the cell membranes of epithelial and endothelial cells (Verkman, 2002; Hara-Chikuma and Verkman., 2006). AQP-1 has recently been described as a potential target for human disease therapies, as AQP-1 knockout mice demonstrated pathological and physiological impacts on cellular proliferation and migration, in addition to defects in the secretion of epithelial fluids and disrupted embryonic development (Hua et al., 2019).

In blood vessels, the function of AQP-1 in permeability processes has been well described, where it acts as a passive water pore that is responsible for the increased water permeability of the cell membrane, driven by osmotic gradient forces (Hara-Chikuma and Verkman, 2006). However, the roles of AQP-1 in SG structures, especially during developmental processes, remains to be determined.

In a previous study, our group identified AQP-1 in myoepithelial and endothelial cells in developing human SGs (de Paula et al, 2017). This finding was supported by other studies, which reported similar finding of AQP-1 in human and rodent glands (Delporte and Steinfield, 2006; Sapmaz et al., 2016). Despite this morphological evidence, only scattered reports have postulated specific functions for AQP-1 in SGs. Galán-Cobo et al. (2016) suggested that AQP1 is important for cell proliferation, as it may regulate the cell volume during the DNA duplication steps of the cell cycle.

AQP-3 is part of the aquaglyceroporin subfamily, transporting glycerol and other small solutes, in addition to water (Agre, 2006; Calamita et al., 2018). In human epithelial cells, AQP3 is considered to be an important protein for the transport and regulation of cellular water volume (Kida et al., 2005). AQP-3 is widely expressed in SGs (Gresz et al., 2001; Larsen et 
al., 2009; Nakamura et al., 2013; Aure et al., 2014; de Paula et al., 2017; Ichiyama et al., 2018). In fully developed human SGs (Gresz et al., 2001), as well as during development (de Paula et al., 2017), AQP-3 is expressed on the basolateral membranes of acinar cells. In mouse embryos, AQP-3 expression has been detected from E13.5, by RT-PCR (Larsen et al., 2009). Similar to AQP-1, the overexpression of AQP-3 has been reported to promote cell cycle progression by stimulating DNA synthesis and regulating cyclins, resulting in increased cell proliferation (Galán-Cobo et al., 2015).

AQP -5 has been identified as the major AQP in SGs, playing an important role in saliva secretion (Delpote et al., 2016) and being primarily expressed in the apical cell membranes of the glandular epithelium (Matsuzaki et al., 2012; Teymoortash et al., 2012). This protein was found to be released in human saliva (Wang et al., 2009) and to regulate the permeability of the acinar plasma membrane (Krane et al., 2001; Delporte and Steinfield, 2006; Larsen et al., 2011), promoting the fundamental balance among electrolytes in secreted salivary fluid (Krane et al., 2001).

Fully developed SGs are known to express AQP-5 in the luminal membranes of acinar cells (Gresz et al., 2001; Matsuki et al., 2005). This pattern could also be observed in the apical acini and intercalated ducts of developing human SGs (de Paula et al., 2017). AQP-5 has also been described during late canalicular stages (E15-E16) of submandibular glands (SMGs) in mouse embryos (Larsen et al., 2011). Aure et al. (2011) have also suggested that AQP-5 plays a role in the embryogenesis of mouse SGs. In rats, AQP-5 was also found in the vascular structures and pro-acinar membranes of developing SGs from E18 (Akamatsu et al, 2003). This protein has been determined to be a positive marker for acinar and pro-acinar cells (Larsen et al., 2011; Hosseini et al., 2018)

The results of previous studies have indicated an expressive participation of AQPs in SGs during developmental stages, although the details of their expression pattern and morphological distribution remain to be determined, in order to better understand their roles during physiology and disease. The present study aimed to perform a detailed morphological investigation of AQP-1, -3 , and -5 in murine developing SGs, describing the specific cellular and physiological expression pattern and how they correlate with associated structures of endothelium (CD31), myoepithelium (SMA) and proliferating cells (PH3).

\section{MATERIALS AND METHODS}

\subsection{Organ Culture}


Wild-type pregnant CD1 mice were collected at different embryonic stages from E13.5 to E16.5. Embryonic submandibular (SMG) and sublingual (SLG) glands were dissected for organ culture and immunofluorescence. All animal procedures were performed in accordance with the guidelines of the UK Home Office at King's College London following the schedule 1 method as per local regulation. Animal experiments conformed with animal research: reporting of in vivo experiments (ARRIVE) guideline, and animals were housed in approved, nonspecific-pathogen-free conditions.

A Leica dissecting stereoscope was used to carefully dissect CD1 mouse embryonic SGs (SMG and SLG), which were removed with the aid of fine tweezers (figure 1 illustrates the dissection sequence). Immediately after dissection, the glands were placed on permeable membrane filters (Cell Culture Insert $0.4 \mu \mathrm{m}, \mathrm{BD}$ ) in dishes containing culture medium (Advanced Dulbecco's Modified Eagle Medium F12, Invitrogen, Waltham, MA, USA; containing $1 \%$ GlutaMAX, Invitrogen, and 1\% penicillin-streptomycin). Embryos were photographed using the same stereoscope.

Samples were cultured at $37^{\circ} \mathrm{C}$ and $5 \% \mathrm{CO}_{2}$, for 1,3 , and 5 days, to obtain precise desired developmental stages. SGs were fixed in $4 \%$ formaldehyde, for $30 \mathrm{~min}$ at room temperature, and washed in phosphate-buffered saline (PBS) before starting the immunofluorescence protocol.

\subsection{Immunofluorescence by Whole Mount}

Whole embryonic salivary gland samples were permeabilised in PBS containing $0.5 \%$ Triton, 6 times for 30 min each wash, and incubated in $1 \times$ Trypsin in PBS on ice, followed by two rinses in PBS for 10 min on ice, and 3 washes of 15 minutes in PBS containing $0.5 \%$ Triton. Specimens were then incubated in blocking solution for 2 hours, under gentle mixing conditions at room temperature, followed by incubation in primary antibodies, at $4^{\circ} \mathrm{C}$ for 48 hours, at concentrations described in Table 1. Excess antibodies were removed by several 30 min washes in PBS containing 0,5\% Triton at room temperature, followed by secondary antibody incubation (1:500 in blocking solution, Alexa Fluor, Invitrogen; 1:1,000 DAPI, Invitrogen) for 2 nights (approximately 48 hours of incubation). Samples were again washed in PBS containing $0.5 \%$ Triton and PBS alone, before being flattened and mounted on glass slides with Fluoroshield (Abcam). Samples were then analysed and imaged using a laser confocal scanning microscope (Leica TCS SP5). 


\section{RESULTS}

\subsection{AQP-1}

Organotypic cultures of embryonic SMGs and SLGs revealed progressive lobular growth during ex vivo incubation. From E13.5 to E16.5, both ductal and acinar branchings showed structural features of pseudoglandular, canalicular, and terminal bud stages, as previously described (Tucker., 2007).

AQP-1 was continuously expressed from E13.5 until E16.5 (Figure 2). During the pseudoglandular (E13.5) and canalicular (E14.5) stages, AQP-1 was observed in scattered cells, throughout the stromal tissue, particularly in vascular structures. In the glandular epithelium, AQP-1 was found in ductal and acinar cells, predominately at the basal pole.

Later on during E15.5 and E16.5, AQP-1 expression was strongly detected in the vascular endothelium, while acinar and ductal cells showed AQP-1 expression patterns similar to those described here during the pseudoglandular and canalicular stages. At E16.5, AQP-1 expression surrounded the luminal cells of the intralobular canalising ductal structure, along the entire lobule thickness (analysed in confocal stacks).

Double-labelling revealed positive cells that were positive for AQP-1 and $\mathrm{PH} 3$, a marker of mitosis (Figure 3). During the late pseudoglandular stage, at E14.5, epithelial SMG and SLG cultures showed cells with nuclear mitotic aspects that co-expressed AQP-1, at the basolateral cellular membranes, and $\mathrm{PH} 3$ in the nucleus. This pattern was maintained during later stages, including the canalicular/terminal bud stage at E16.5, during which AQP-1 was observed to co-express with $\mathrm{PH} 3$ in numerous acinar and ductal cells.

Similar expression patterns were observed for AQP-1, CD31, and SMA in the SMG and SLG.

Distinct portions of the endothelium were identified by each endothelial marker. At E13.5, double staining with AQP-1 and SMA revealed a vascular architecture widespread along the tissue (Figure 4A).

At E15.5, the glandular architecture had developed a complex vascular network. AQP-1 expression was detected in several vascular portions, whereas SMA was identified in different endothelial tissue, with only occasional overlap (Figure 4B). At E16.5, with glandular developmental progression, AQP-1 exhibited vascular expression, whereas SMA became concentrated in presumptive myoepithelial cells (Figure 4C). 
Double staining of AQP-1 and CD31 revealed the expression of CD31 in endothelial cells, whereas AQP-1 was localised in different vascular portions (Figure 4D).

\section{$\underline{3.2 \mathrm{AQP}-3}$}

AQP-3 was identified in both the SMG and SLG epithelium and was primarily observed in mesenchymal tissue, from E13.5 to E16.5 (Figure 5). AQP-3 was found in the basolateral membranes of some scattered cells, which were also PH3-positive, at E13.5 (Figure 5A-A", arrowheads). At E14.5 and E15.5, PH3 was intensely observed, especially in AQP-3expressing cells. At E16.5, when the glandular tissue acquired well-developed morphological characteristics, $\mathrm{PH} 3$ expression declined and AQP-3 protein was confined to specific glandular tissues, such as the oral mucosa, and the promimal ductal portions of the gland, previously reported (de Paula et al., 2017).

Double-labelling of AQP-3 and CD31 revealed no overlap between these markers during the various SG development phases examined in this study. At E16.5, an intricate and well-established vascular network was revealed by CD31 staining, in the mesenchymal endothelium (Figure 6, arrows).

A strong AQP-3 expression pattern was observed in basolateral cell membranes, and some structures exhibiting AQP-3 positive cells, believed to be endothelial tissue, were also identified within the mesenchyme (Figure 6, arrowheads).

\section{$\underline{3.3 \mathrm{AQP}-5}$}

AQP-5 was not identified during the very early developmental stages analysed in this study (E13.5). This protein was weakly positive at E14.5, in the epithelial cellular membranes of pro-acinar structures (Figure 7A and A", arrowhead). AQP-5 expression was increasingly detected at E15.5 and E16.5, in luminal intercalated portions, primarily confined to the apical membranes of acinar and ductal structures (Figure 7B and B", arrowhead and C and C", respectively). At these stages, $\mathrm{PH} 3$-positive cells were identified in distinct areas where AQP5 was positive (Figure 7B", arrow). 


\section{DISCUSSION}

AQP-1, -3, and -5 proteins have been previously described during mouse SG development (Larsen et al., 2009), however their morphological expression and relationship with stromal tissues have not been fully explored. Here, the results illustrated a detailed temporal and morphological expression of the main types of aquaporin during murine SG development and their association with myoepithelial, vascular and proliferation markers at the cellular level.

Previously, AQP-1 positive cells were suggested to be myoepithelial cells, however our analysis suggests that they are more likely to be vascular cells that are closely asscoiated with acinar areas. In addition, the expression of AQP-1 and AQP-3 were in turn found to label many proliferating cells during initial stages of gland development, agreeing with previous studies that suggested a role in the cell cycle (Galán-Cobo et al., 2015, 2016). AQP-5 in turn showed a marked expression during late stages of development within ducts and apical acinar areas and did not overlap significantly with the pattern of proliferation.

AQP-1 is a water channel that has previously been shown to be expressed during rat (Akamatsu et al., 2003), mouse (Larsen et al., 2009), and human (de Paula et al., 2017) SG development. Here, we found that AQP-1 was predominantly present in

the glandular parenchyma and mesenchymal blood vessels during early SG development, which was similar to that previously described during early human SGs development (de Paula et al., 2017). Morphologically in the gland epithelium, AQP-1 appears to have a distinct distribution pattern in both developing and fully developed SGs. Our study identified cellular basal membranes expressing AQP-1 starting at E13.5, not just in vascular tissue but also in incipient ductal and acinar cells that form the SG epithelium, at all analysed stages.

We also showed that AQP-1 was present along the developing vasculature evidenced by SMA co-expression, suggesting the importance of this molecule for both gland epithelium and stromal tissues from early morphogenesis. AQP-1 expression has been described in capillaries and vasculature during the development of the rat SMG, but not in acinar or ductal cells (Akamatsu et al., 2003). These different findings regarding AQP-1 protein expression patterns may be due to differences in the models analysed or the method applied. The striking vascular expression suggests that AQP-1 function may be crucial during initial glandular morphogenesis and vascular development necessary to nurture the glandular parenchyma.

The role played by AQP-1 in the microvasculature is not yet fully understood. Nakamura and colleagues (2013) identified AQP-1 in blood vessels and myoepithelial cells in the SGs of 
adult mice (Nakamura et al., 2013). Akamatsu and colleagues (2003) reported the constitutive expression of $A Q P 1$ mRNA during development, whereas only a weak expression pattern was detected in the adult rat SG (Akamatsu et al., 2003), which suggested that this protein may be important for developmental mechanisms, but less for homeostasis. In the adult mouse SMG and SLG, AQP-1 expression has been described in endothelial cells, and AQP1 mRNA was more strongly detected in the SLG than in the SMG. In addition, higher AQP1 levels have been reported in male SMG in comparison to females (Aure et al., 2014).

Although AQP-1 expression in vascular tissue has been widely described in the literature, no altered saliva production was identified in conditional $A Q P 1$ knockout mice, when $A Q P 1$ was deleted from the microvascular endothelium (Ma et al., 1999). AQP1 null mice, in contrast, revealed impaired cell migration and altered vessel formation (Saadoun et al., 2005). In this study, AQP-1 staining was observed in endothelial tissue within the glandular stroma. Double staining for CD31 and AQP-1, showed however no co-expression along the vascular tissue, with clear overlapping of different blood vessels demonstrating completely distinct expression. In contrast, staining for SMA, which has also been described as an endothelial marker during human SG development (lanez et al., 2010), was observed to overlap with AQP1 in some large endothelial structures, from early development (E13.5).

AQP-1 has also been described as involved in the migration of tumour cells in mice $\mathrm{Hu}$ and Verkman, 2006); however, the physiological role of AQP in proliferative cells remains controversial. Most recently, proliferation associated molecules that are present in the cell cycle, including cyclins, were found to be abnormal by concomitant AQP-1 expression (GalánCobo et al., 2016). In addition, the levels of $A Q P 1$ mRNA and protein expression have been described to fluctuate during different phases of the cell cycle, with reduced cellular expression levels during phases S and G2/M and increasing during G0/G1 (Delporte et al., 1996). In SMG and SLG stromal or epithelial tissues, AQP-1 immunoreactivity was primarily identified in cells characterised by nuclear mitosis properties, as evidenced by the coexpression of AQP1 and the proliferative cell marker PH3. AQP-1 may, therefore, act as a proliferative moderator and facilitating mitosis.

Here, AQP-3 results also reveal an important presence in vasculature during SG development, and its presence in these structures may facilitate AQP-1 function, as blood supply is fundamental during tissue growth. Previous report showed AQP-3 is expressed during cell proliferation and migration (Hara-Chikuma and Verkman, 2008).

Our present results revealed that most AQP-3-positive cells are involved in mitotic processes (PH3-positive). These findings contribute new information to the findings reported 
by Nakamura and colleagues (2013), who observed the presence of AQP-3 in the basolateral membranes of acinar cells and the ductal membranes and cytoplasm of the mouse SG.

Additionally, Akamatsu et al. (2003) described the expression of AQP3 mRNA in the rat developing SMGs, suggesting that this molecule could play a role in SG development. Furthermore, our results revealed AQP-3 expression in mesenchymal structures, which were interpreted as blood vessels. However, CD31 and AQP-3 did not show colocalisation in the assays performed in this study. We speculate that AQP-3-positive/CD31-negative vascular structures may represent immature blood vessels during the process of development; however, this speculation requires further confirmation. Alternatively, these structures may represent lymphatic vessels, as SGs contain an active network of lymphatic vessels that participate in the regulation and balance of interstitial fluids (Breslin et al., 2018). The lymphatic fluids in these vessels, among other substrates, contain glycerol, which is transported through AQP-3 channels (Hara-chikuma and Verkman, 2005).

One of the most relevant and well-studied water channels in SGs is AQP-5. This work revealed an important AQP-5 expression in several intraductal regions, specifically during late developmental stages (E16.5). This result agrees with previous findings, which described AQP-5 expression in mature SGs in diverse mammalian models (Ma et al., 1999; Matsuzaki et al., 1999; Gresz et al., 2001; Krane et al., 2001). During human SG development, AQP-5 was also observed in specific portions of the intercalated ductal lumen, corroborating the results presented here (de Paula et al., 2017). Interestingly, although the tissue expression of AQP-5 has only been detected in SGs during canalicular stages in this study, AQP5 mRNA has been identified earlier at E13.5, with increasing levels through birth (Larsen et al., 2009; Aure et al., 2011). The late tissue expression may reflect the active status of the protein, which has also been identified in structures including blood vessels and nerves fibres in SGs (Akamatsu et al., 2003).

In summary, our results revealed the specific expression patterns of AQPs during mouse SMG and SLG development. AQP-1 and -3 were primarily expressed in mitotic cells during initial SMG and SLG development, whereas AQP-5 was expressed during the late stages of SG development, likely as an important molecule during the process of saliva formation.

\section{ACKNOWLEDGEMENTS}


This research was funded by Coordenação de Aperfeiçoamento de Pessoal de Nível Superior (CAPES), Programa de Doutorado Sanduíche no Exterior (PDSE), process number 88881.188920/2018-01. We thank Lisa Giles, PhD, from Blue Pencil Science (http://www.bluepencilscience.com/) for editing an English draft of this manuscript.

\section{AUTHOR CONTRIBUTION}

Conceptualisation: S.V.L., A.S.T., and M.M.S.N.; formal analysis: F.P., A.S.T., T.H.N.T., and S.V.L.; investigation: F.P., T.H.N.T., M.M.S., and C.M.C.C.; resources: S.V.L. A.S.T., and M.M.S.N; writing-original draft preparation: F.P. S.V.L., and T.H.N.T.; writing-review and editing: C.M.C.C. and S.V.L.; visualisation: S.V.L. and F.P.; supervision: S.V.L., A.S.T., and M.M.S.N.; project administration: S.V.L.; funding acquisition: S.V.L.

\section{DATA AVAILABILITY STATEMENT}

All data generated by this study are included in the manuscript and accompanying supplementary material.

\section{ORCID}

Fernanda de Paula https://orcid.org/0000-0002-4214-1149

\section{REFERENCES}

Agre P. (2006) The Aquaporin Water Channels. Proc Am Thorac Soc, 3, 5-13.

Akamatsu T, Parvin MN, Murdiastuti K, Kosugi-Tanaka C, Yao C, Miki O, Kanamori N, Hosoi K. (2003) Expression and localization of aquaporins, members of the water channel family, during development of the rat submandibular gland. Pflugers Arch, 446, 641-51.

Aure MH, Ruus AK, Galtung HK. (2014) Aquaporins in the adult mouse submandibular and sublingual salivary glands. J. Mol. Histol, 45, 69-80.

Aure MH, Larsen HS, Ruus AK, Galtung HK. (2011) Aquaporin 5 distribution pattern during development of the mouse sublingual salivary gland. J Mol Histol, 42, 401-8. 
Breslin JW, Yang Y, Scallan JP, Sweat RS, Adderley SP, Murfee WL. (2018) Lymphatic Vessel Network Structure and Physiology. Compr Physiol, 13;9, 207-99.

Calamita G, Perret J, Delporte C. (2018) Aquaglyceroporins: drug targets for metabolic diseases? Front Physiol, 10;9:851.

Chauvigné F, Yilmaz O, Ferré A, Fjelldal PG, Finn RN, Cerdà J. (2019) The vertebrate Aqp14 water channel is a neuropeptide-regulated polytransporter. Commun Biol, ;2(1):462.

de Paula F, Teshima THN, Hsieh R, Souza MM, Coutinho-Camillo CM, Nico MMS, Lourenco SV. (2017) The expression of water channel proteins during human salivary gland development: a topographic study of aquaporins 1, 3 and 5. J Mol Histol, 48, 329-336.

Delporte C, Chen ZJ, Baum BJ. (1996) Aquaporin 1 Expression during the Cell Cycle in A5 Cells. Biochem. Biophys. Res. Commun, 228, 223-8.

Delporte C, Steinfeld S. (2006) Distribution and roles of aquaporins in salivary glands. Biochim. Biophys. Acta, 1758, 1061-70.

Delporte C, Bryla A, Perret J. (2016) Aquaporins in Salivary Glands: From Basic Research to Clinical Applications. Int J Mol Sci. 27;17(2).

Galán-Cobo A, Ramírez-Lorca R, Serna A, Echevarría M. (2015) Overexpression of AQP3 Modifies the Cell Cycle and the Proliferation Rate of Mammalian Cells in Culture. PLoS One, $14 ; 10(9)$.

Galán-Cobo A, Ramírez-Lorca R, Toledo-Aral JJ, Echevarría M. (2016) Aquaporin-1 plays important role in proliferation by affecting cell cycle progression. J. Cell. Physiol, 231, 243-56.

Gresz V, Kwon TH, Hurley PT, Varga G, Zelles T, Nielsen S, Case RM, Steward MC. (2001) Identification and localization of aquaporin water channels in human salivary glands. Am J Physiol Gastrointest Liver Physiol, 281, G247-54.

Hara-Chikuma M, Verkman AS. (2006) Aquaporin-1 facilitates epithelial cell migration in kidney proximal tubule. J. Am. Soc. Nephrol, 17, 39-45.

Hara-Chikuma M, Takahashi K, Chikuma S, Verkman AS, Miyachi Y. (2009) The expression of differentiation markers in aquaporin-3 deficient epidermis. Arch Dermatol Res, 301, 245-52. Hara-Chikuma M, Verkman AS. (2005) Aquaporin-3 functions as a glycerol transporter in mammalian skin. Biol Cell, 97, 479-86.

Hara-Chikuma M, Verkman AS. (2008) Aquaporin-3 facilitates epidermal cell migration and proliferation during wound healing. J Mol Med (Berl), 86, 221-31 
Hosseini ZF, Nelson DA, Moskwa N, Sfakis LM, Castracane J, Larsen M. (2018) FGF2dependent mesenchyme and laminin-111 are niche factors in salivary gland organoids. J Cell Sci, 20;131(4).

Hua Y, Xinxin Ying, Yiyu Qian, Haibin Liu, Yehui Lan, Ailan Xie, Xueqiong Zhu. (2019) Physiological and pathological impact of AQP1 knockout in mice. Biosci. Rep, 14;39(5).

$\mathrm{Hu}$ J, Verkman AS. (2006) Increased migration and metastatic potential of tumor cells expressing aquaporin water channels. FASEB J, 20, 1892-4.

lanez RF, Buim ME, Coutinho-Camillo CM, Schultz R, Soares FA, Lourenço SV. (2010) Human salivary gland morphogenesis: myoepithelial cell maturation assessed by immunohistochemical markers. Histopathology, 57, 410-7.

Ichiyama T, Nakatani E, Tatsumi K, Hideshima K, Urano T, Nariai Y, Sekine J. (2018) Expression of aquaporin 3 and 5 as a potential marker for distinguishing dry mouth from Sjögren's syndrome. J Oral Sci, 60, 212-20.

Kida H, Miyoshi T, Manabe K, Takahashi N, Konno T, Ueda S, Chiba T, Shimizu T, Okada Y, Morishima S. (2005) Roles of aquaporin-3 water channels in volume-regulatory water flow in a human epithelial cell line. J Membr Biol, 208, 55-64.

King LS, Nielsen S, Agre P. (1997) Aquaporins in complex tissues. I. Developmental patterns in respiratory and glandular tissues of rat. Am. J. Physiol, 273, C1541-8.

Krane CM, Melvin JE, Nguyen HV, Richardson L, Towe JE, Doetschman T, Menon AG. (2001) Salivary acinar cells from aquaporin 5-deficient mice have decreased membrane water permeability and altered cell volume regulation. J Biol Chem, 276, 23413-20.

Larsen HS, Ruus AK, Galtung HK. (2009) Aquaporin expression patterns in the developing mouse salivary gland. Eur. J. Oral Sci, 117, 655-62.

Larsen HS. Aure MH, Peters ASB, Larsen M, Messelt EB, Galtung HK. (2011) Localization of AQP5 during development of the mouse submandibular salivary gland. J Mol Hist, 42, 71-81.

Ma T, Song Y, Gillespie A, Carlson EJ, Epstein CJ, Verkman AS. (1999) Defective secretion of saliva in transgenic mice lacking aquaporin-5 water channels. J. Biol. Chem, 274, 20071-4. Matsuki M, Hashimoto S, Shimono M, Murakami M, Fujita-Yoshigaki J, Furuyama S, Sugiya $\mathrm{H}$. (2005) Involvement of aquaporin-5 water channel in osmoregulation in parotid secretory granules. J Membr Biol, 203, 119-26.

Matsuzaki T, Suzuki T, Koyama H, Tanaka S, Takata K. (1999) Aquaporin-5 (AQP5), a water channel protein, in the rat salivary and lacrimal glands: immunolocalization and effect of secretory stimulation. Cell Tissue Res, 295, 513-21. 
Matsuzaki T, Susa T, Shimizu K, Sawai N, Suzuki T, Aoki T, Yokoo S, Takata K. (2012) Function of the membrane water channel aquaporin-5 in the salivary gland. Acta Histochem Cytochem. 45, 251-9.

Nakamura M, Saga T, Watanabe K, Takahashi N, Tabira Y, Kusukawa J, Yamaki K. (2013) An immunohistochemistry-based study on aquaporin (AQP)-1, 3, 4, 5 and 8 in the parotid glands, submandibular glands and sublingual glands of Sjögren's syndrome mouse models chronically administered cevimeline. Kurume Med J, 60, 7-19.

Qin Y, Wang S, Duan X, Liu D. (2019) Expression analysis of the aquaporins during zebrafish embryonic development. Gene Expr Patterns, 32, 38-43.

Saadoun S, Papadopoulos MC, Hara-Chikuma M, Verkman AS. (2005) Impairment of angiogenesis and cell migration by targeted aquaporin-1 gene disruption. Nature, 434, 78692.

Sapmaz E, Uysal M, Tumer MK, Sapmaz HI, Somuk BT, Arici A, Tas U. (2016) Investigation of age-related changes in the expression of aquaporin- 1 and aquaporin- 5 in the salivary glands of mice. Acta Otolaryngol, 136, 937-43.

Soyfoo MS, Chivasso C, Perret J, Delporte C. (2018) Involvement of Aquaporins in the Pathogenesis, Diagnosis and Treatment of Sjögren's Syndrome. Int J Mol Sci, 30;19(11).

Teymoortash A, Wiegand S, Borkeloh M, Bette M, Ramaswamy A, Steinbach-Hundt S, Neff A, Werner JA, Mandic R. (2012) Variations in the expression and distribution pattern of AQP5 in acinar cells of patients with sialadenosis. In Vivo, 26, 951-5.

Verkman AS. (2002) Aquaporin water channels and endothelial cell function. J. Anat, 200, 61727.

Wang W, Hart PS, Piesco NP, Lu X, Gorry MC, Hart TC. (2003) Aquaporin expression in developing human teeth and selected orofacial tissues. Calcif. Tissue Int, 72, 222-7.

Wang D, Iwata F, Muraguchi M, Ooga K, Ohmoto Y, Takai M, Mori T, Ishikawa Y. (2009) Correlation between salivary secretion and salivary AQP5 levels in health and disease. J Med Invest. 56 Suppl:350-3.

Wellner RB, Redman RS, Swaim WD, Baum BJ. (2006) Further evidence for AQP8 expression in the myoepithelium of rat submandibular and parotid glands. Pflugers Arch, 451, 642-5.

Yang B, Song Y, Zhao D, Verkman AS. (2005) Phenotype analysis of aquaporin-8 null mice. Am. J. Physiol. Cell. Physiol, 288, C1161-70. 
Figure and Table Legends

Table 1. Primary antibodies, clone, manufacturer and titrations employed for immunofluorescence assays.

\begin{tabular}{|c|c|c|c|}
\hline Primary serum & Clone & Manufacturer & Titer \\
\hline $\begin{array}{c}\text { Anti-Aquaporin 1 } \\
\text { (AQP-1) }\end{array}$ & $\begin{array}{c}\text { Mouse } \\
\text { Monoclonal }\end{array}$ & $\begin{array}{c}\text { Abcam } \\
\text { ab9566 }\end{array}$ & $1: 300$ \\
\hline $\begin{array}{c}\text { Anti-Aquaporin 3 } \\
\text { (AQP-3) }\end{array}$ & $\begin{array}{c}\text { Mouse } \\
\text { Monoclonal }\end{array}$ & $\begin{array}{c}\text { Santa Cruz } \\
\text { SC-518001 }\end{array}$ & $1: 200$ \\
\hline $\begin{array}{c}\text { Anti-Aquaporin 5 } \\
\text { (AQP-5) }\end{array}$ & $\begin{array}{c}\text { Rabbit } \\
\text { polyclonal }\end{array}$ & $\begin{array}{c}\text { Sigma-Aldrich } \\
178615\end{array}$ & $1: 200$ \\
\hline $\begin{array}{c}\text { Phosho-Histone H3 } \\
\text { (PH3) }\end{array}$ & $\begin{array}{c}\text { Rabbit } \\
\text { Polyclonal }\end{array}$ & $\begin{array}{c}\text { Cell Signaling } \\
\# 9701\end{array}$ & $1: 500$ \\
\hline $\begin{array}{c}\text { Smooth Muscle } \\
\text { Actin }\end{array}$ & Rabbit & Abcam \\
(SMA) & Polyclonal & ab5694 & $1: 200$ \\
\hline CD-31 & Rabbit & Abcam & ab28364 \\
\hline
\end{tabular}


Table 2. Summary of Results

\begin{tabular}{|c|c|c|c|c|c|c|c|c|}
\hline Stages & $\begin{array}{l}\text { Morphological aspects } \\
\text { (SMG) }\end{array}$ & Structures & AQP-1 & AQP-3 & AQP-5 & $\mathrm{PH} 3$ & CD31 & SMA \\
\hline \multirow{4}{*}{$\begin{array}{c}\text { Pseudo } \\
\text { glandular } \\
\text { E13.5 }\end{array}$} & \multirow{4}{*}{$\begin{array}{l}\text { Initial branching } \\
\text { morphogenesis } \\
\text { approximately } \\
4-5 \text { forming buds }\end{array}$} & Epithelial & ++ & + & - & + & 0 & - \\
\hline & & $\begin{array}{c}\text { Duct } \\
\text { formation }\end{array}$ & ++ & + & - & + & 0 & - \\
\hline & & Vasculature & + & + & - & + & 0 & +++ \\
\hline & & $\begin{array}{l}\text { Myoepithelial } \\
\text { cells }\end{array}$ & - & - & - & - & 0 & - \\
\hline \multirow{4}{*}{$\begin{array}{c}\text { Canalicular } \\
\text { E14.5 - } \\
\text { E15.5 }\end{array}$} & \multirow{4}{*}{$\begin{array}{c}\text { Epithelial cells } \\
\text { proliferating/ } \\
\text { multi-lobed gland } \\
\text { Ducts developing lumen }\end{array}$} & Epithelial & ++ & + & ++ & ++ & 0 & - \\
\hline & & $\begin{array}{c}\text { Duct } \\
\text { formation }\end{array}$ & ++ & + & + & ++ & 0 & - \\
\hline & & Vasculature & ++ & - & - & ++ & 0 & +++ \\
\hline & & $\begin{array}{l}\text { Myoepithelial } \\
\text { cells }\end{array}$ & - & - & - & - & 0 & ++ \\
\hline \multirow{4}{*}{$\begin{array}{l}\text { Terminal } \\
\text { Bud } \\
>\mathrm{E} 16.5\end{array}$} & \multirow{4}{*}{$\begin{array}{l}\text { Branches and terminal } \\
\text { buds voiding to form } \\
\text { well-structured acini and } \\
\text { ducts }\end{array}$} & Epithelial & ++ & ++ & +++ & +++ & - & - \\
\hline & & $\begin{array}{c}\text { Duct } \\
\text { formation }\end{array}$ & ++ & + & ++ & +++ & - & - \\
\hline & & Vasculature & +++ & - & - & - & +++ & +++ \\
\hline & & $\begin{array}{l}\text { Myoepithelial } \\
\text { cells }\end{array}$ & ++ & - & - & - & - & +++ \\
\hline
\end{tabular}

*Intensity of immunofluorescence stain: weak (+), mild (++), strong (+++), negative (-), (0) not tried.

Figure 1: Embryonic CD1 mouse cord sequence, just after cesarean sectioning (A). E13.5 CD1 mouse embryo, inside the placenta. (B) Tongue dissection and withdrawal, showing the lateral sectioning of the SMG and SLG (C).

Figure 2: AQP-1 expression pattern during CD1 mouse submandibular gland (SMG) development.

A: E13.5 (pseudoglandular stage): AQP-1 staining at the cellular basal membrane (green), in a scattered pattern over the glandular buds (arrow), along the epithelial stalk (arrowhead), and in endothelial tissue (asterisks). 
B: Canalicular stage morphology at E14.5: AQP-1 expression (green) in buds (arrow) and microvasculature (asterisks);

C: Late canalicular stage morphology at E15.5: AQP-1 in the cellular basal pole (green), in buds (arrow) and ducts (arrowhead). The protein is also detected in the stromal microvasculature (asterisks).

D: Early terminal bud stage (E16.5): strongly expressing AQP-1 (green) in well-established endothelial tissue surrounding the SG (asterisks) and surrounding the cellular basal pole, in a scattered pattern, in buds (arrow) and ducts (arrowhead).

\section{Figure 3: AQP-1 in mouse developing salivary glands}

A: AQP-1 expression in basal cell membranes (green - A') co-expressed with $\mathrm{PH} 3$ nuclear staining (red - A").

B: At E14.5: AQP-1 and PH3, showing expression in the ductal and acinar epithelium.

C and D: Salivary gland at E16.5, showing condensed mitotic nuclei (blue) co-localised with AQP-1 in several acinar cells and ductal epithelia.

Figure 4: co-expression of AQP-1 and SMA in mouse developing salivary glands

A: E13.5 double labelling, showing AQP-1 (green) and SMA (red), in overlapping vascular tissue over the endothelium.

B: E15.5 double labelling, revealing AQP-1 (green) and SMA (red) in colocalised in some microvasculature sites and the single labelling of each marker in endothelial tissue.

C: E16.5 (late canalicular/terminal bud stage): AQP-1 expression in endothelial structures (green), with SMA-positive cells surrounding the epithelial bud staining (presumptive myoepithelial cells).

D: Microvasculature expression of AQP-1 (red), and in the basal aspect of the acinar epithelium (green); CD31 (red) at different sites, overlapping the endothelium.

Figure 5: co-expression of AQP-3 and PH-3 in mouse developing salivary glands

A: AQP-3 expression in basal cell membranes (green - A'), expressed with PH3 nuclear stain (red - A").

B: At E14.5: AQP-3 and PH3, showing expression in the ductal and acinar epithelium (B-B").

C: At E15.5: Strong AQP-3 (C) expression and PH3 (C'), distributed in mesenchyme cells, showing the co-expression of AQP-1 and nuclear PH3 in basolateral cell membranes (C").

D: E16.5: AQP-3 expression and intense PH3 expression in mesenchyme cells (D-D").

Figure 6: co-expression of AQP-3, CD31 and SMA in mouse developing salivary glands

E16.5 double staining, with CD31 and AQP-3. CD31 (green) is observed in an intricate microvascular network, surrounding SMG structures. AQP-3 expression was detected in basolateral cell membranes (red) and suspected microvasculature tissue over the mesenchyme (arrowhead).

Figure 7: co-expression of AQP-5 and PH3 in mouse developing salivary glands 
At E14.5, AQP-5 protein expression was barely observed in pro-acini structures over the glandular epithelium (A", arrowhead); at this stage, some apoptotic cells were identified by lysotracker (white). At E15.5, the expression of AQP-5 showed staining in acinar apical membranes (B", arrowhead) and independent PH3 expression (B', arrow). At E16.5, intense AQP-5 expression was observed in apical acinar buds (green) and PH3 expression (red) was observed at different sites over the glandular epithelium. 
